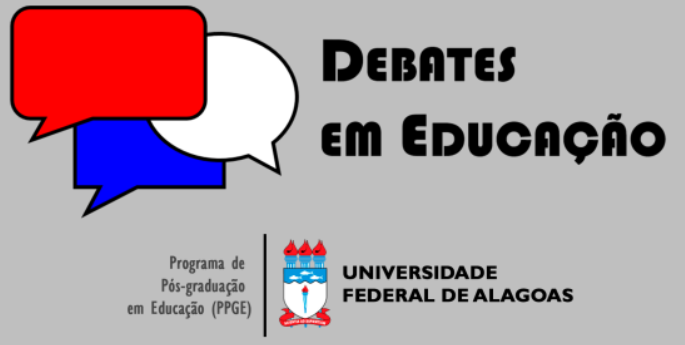

ISSN Eletrônico 2175-6600

Vol. 12 | No. 26 | Jan./Abr. | 2020

\section{Glenda Galeotti}

Università degli Studi di Firenze (UNIFI - Itália) glendagaleotti@unifi.it

\section{ARTESANATO E TRADIÇÃO NO SUL DA ITÁLIA: PATRIMÔNIO CULTURAL, NOVAS HABILIDADES E DESENVOLVIMENTO SUSTENTÁVEL'}

\section{RESUMO}

O texto apresenta uma pesquisa sobre artesanato artístico e tradicional no sul da Itália, que visa identificar produções artesanais de excelência em risco de extinção, mas que mostram potencial de desenvolvimento. A investigação centra-se no reconhecimento das produções artesanais como patrimônio cultural intangível de uma comunidade local, na emergência do seu potencial para o desenvolvimento sustentável, no fortalecimento do diálogo, da aprendizagem e da solidariedade entre gerações, entre mestres de arte e jovens talentos pela refuncionalização e atualização da produção. Os resultados da pesquisa foram utilizados para desenvolver três intervenções de empreendimentos sociais, que assumo como objeto de suas produções artísticas e tradicionais.

Palavras-chave: Educação intergeracional. Saberes tradicionais. Áreas marginais. Solidariedade social.

\section{ARTISANSHIP AND TRADITION IN SOUTHERN ITALY: CULTURAL HERITAGE, NEW SKILLS AND SUSTAINABLE DEVELOPMENT}

\begin{abstract}
The text presents a research on artistic and traditional artisanship in southern Italy, which aims to identify artisanal productions of excellence in danger of extinctions, but which show potential for development. The research focuses on the recognition of artisanal productions as an intangible cultural heritage of a local community, the emergence of their potential for sustainable development, the strengthening of intergenerational dialogue, learning and solidarity, between art masters and young talents through refunctionalization and updating of production. The research results were used to develop three interventions for the creation of social enterprises, which I assume as object of their artistic and traditional productions.
\end{abstract}

Keywords: Intergenerational education. Traditional knowledge. Marginal areas. Social solidarity.

Submetido em: 28/07/2019

Aceito em: 25/1।/2019

Publicado em: 06/04/2020

do http://dx.doi.org// 0.28998/2175-6600.2020v12n26p485-499 


\section{PREMISSA}

Este artigo ilustra uma pesquisa nacional realizada como parte da iniciativa "Artesanato e Tradição no Sul: patrimônio e crescimento de novas habilidades", do Observatório dos Mestres de Arte de Florença. A pesquisa visa identificar atividades artesanais tradicionais, em risco de extinção em seis regiões do sul da Itália, mas ao mesmo tempo evidencia o potencial de desenvolvimento para as áreas em que estão localizadas. Em suma, trata-se de identificar quais produções artesanais, se adequadamente apoiadas por ações sistêmicas, podem representar uma oportunidade para o desenvolvimento social e econômico de áreas estagnadas ou em depressão.

Partindo dessas considerações, o trabalho apresentado aqui se desenvolve a partir de uma definição de artesanato como um processo de transformação do ambiente físico e natural no qual o ser humano está inserido e através do qual desenvolve conhecimentos específicos, habilidades e visões de mundo, que são a espinha dorsal dos sistemas de vida de uma dada comunidade.

De acordo com a perspectiva ecológica (BRONFENBRENNER, 1979), a atividade artesanal pode representar uma das modalidades relacionais que os seres humanos estabelecem com o meio ambiente e demonstra como essa relação evolui através de uma experiência específica e determinada de habitar o ambiente, de maneira ativa, operacional e coletiva (BATESON, 1973, 1979).

O ambiente, de fato, não é uma realidade neutra em que o sujeito projeta ideias e representações, mas um mundo entrelaçado com a própria vida dos indivíduos que ali se enraizam, integrados (embutidos) na experiência de serem corpos específicos em um ambiente específico, este útlimo entendido tanto em seu componente biofísico quanto como um conjunto de elementos sociais, técnicos e culturais (INGOLD, 2004, 2009). Ao mesmo tempo, o meio ambiente e seus recursos, como produtos sociais historicamente ativados, são definidos, em sua ecologia, pelas práticas de controle, produção e conhecimento das sociedades humanas que os utilizaram ao longo do tempo. $\bigcirc$ ambiente natural, biológico e físico em que o ser humano está imerso não é propriamente criado, mas profundamente modificado e, portanto, construído pela ação humana. $\bigcirc$ artesanato pode, portanto, ser considerado uma ação de modificação e construção do meio ambiente, através da qual conhecimentos e habilidades são desenvolvidos, mas também significados através de visões de mundo. Esse know-how, na verdade, não considera apenas habilidades técnicas, uma vez que é composto por uma série de percepções altamente subjetivas, representadas por sensações sutis que são o resultado de uma prática constante ao longo do tempo, no espaço e de processos coordenados entre várias partes do corpo, ferramentas de trabalho e objetos ou materiais trabalhados.

Conhecimentos rotineiros e sistemáticos, conhecimento embutido, conhecimento dificilmente automatizável e formalizável, na medida em que são fruto da aprendizagem experiencial e manifestam-se 
através de comportamentos complexos "vividos" pelo corpo, conhecimento incorporado, conhecimento imediatamente compreendido por aqueles que compartilham os mesmos referenciais de significado, conhecimento encultural. Esses são conceitos que descrevem o conhecimento artesanal e referem-se às formas situadas e contextuais do conhecer (VASSALLO, 2003).

Esses conhecimentos são objeto de percursos de formação predominantemente informais, que se desenvolvem em locais de trabalho e oficinas artesanais. Os processos e os mecanismos que permitem a passagem de conhecimentos e habilidades estão no centro de estudos e pesquisas que investigam os processos de formação desenvolvidos dentro das comunidades e na relação entre mestres e aprendizes. Entre outros, o conceito de "comunidade de práticas" por Etienne Wenger, encontrou grande sucesso devido à sua capacidade heurística e interpretativa da relação educativa que se instaura entre aqueles que compartilham um interesse e um código comum. A comunidade de prática é um sistema auto-organizado que se desenvolve em três dimensões:

- campos temáticos: agrupam os membros que participam e podem evoluir;

- comunidade: elemento que estimula o compartilhamento de ideias e interações;

- prática: conhecimento específico que é compartilhado e mantido.

Wenger juntamente com Jean Lave (1990), indagam os aspectos sociais e situados da aprendizagem que ali se desenvolve. Veicula significados daquele contingente, com respeito aos propósitos práticos aos quais se dirige, sendo, portanto, emocional e corpóreo, dependente também da linguagem, de formas específicas de comunicação e participação em uma comunidade, entendida como uma multiplicidade de interesses e de relações de poder (GHERARDI, 2008).

As comunidades de práticas também podem ser lidas como dispositivos de educação intergeracional, que favorecem a comunicação e a compreensão entre diversas gerações através da aprendizagem recíproca: isso se torna um meio de sociabilidade e coesão, uma vez que está exclusivamente orientado para o desenvolvimento de habilidades técnico-práticas.

trabalho não tem apenas implicações pessoais, mas também sociais, uma vez que ocorre em um contexto comunitário de solidariedade, no qual é oferecida a oportunidade de os indivíduos se expressarem e crescerem. Esse vínculo é condição indispensável para garantir, na sociedade globalizada contemporânea, o valor e a dignidade do homem na alteridade do encontro e do intercâmbio com o outro. Somente recuperando essa dimensão, o trabalho não reduz seu propósito no objeto que produz, mas, ao contrário, eleva seu significado gerativo em uma perspectiva intesubjetiva e política (COSTA, 20II). 
As acelerações, modificações e mutações contínuas que caracterizam o mundo contemporâneo, muitas vezes colocam o modelo de aprendizagem fora do cotidiano e, portanto, a experiência de mestres artesãos em crise. Torna-se cada vez mais difícil assumir a herança da geração anterior, entendida como acúmulo de conhecimento a ser transferido para gerações futuras, para aprendizes nas oficinas. $\bigcirc$ mesmo sistema de ações para aprender, como tradicionalmente concebido nas oficinas de artesãos da Renascença, estará perdido. Saberes de mulheres e homens artesãos, construídos na vida social, constantemente postos à prova, verificados, transformados, encontram-se hoje diante de um grande desafio, ligado à sua transmissão intergeracional (SENNET, 2008).

Nesse contexto, é necessário partir do desenvolvimento de uma relação autenticamente educacional entre artesãos jovens e sêniors, consistente com a necessidade de integrar a tradição com a modernidade, que oferece numerosas e contínuas oportunidades de inovação. Aprender fazendo não significa transmitir conceitos e categorias com os quais se molda o material disforme da experiência sensorial, mas sim ter a possibilidade de descobrir-se dentro de contextos e situações dadas, para chegar a uma compreensão prática, ao invés da mera aquisição de conhecimento.

A educação intergeracional é, portanto, um exercício social que, convidando à escuta, à construção comum e original, à participação e à integração, pode converter-se em troca, oportunidade de reciprocidade, dimensão de experimentar e descobrir o valor de saber cooperar ativamente (BASCHIERA, DE LUIGI, LUPPI, 20 I4). Isso requer apresentar-se ao outro em uma posição de igualdade porque, como assinala Freire (2004, p. 96): "se me sinto superior ao diferente, não importa quem ele é, me recuso a ouvi-lo ou ouvi-la [...]. Se a estrutura do meu pensamento é o único que considero justo, irrepreensível, não posso ouvir aqueles que pensam ou elaboram seu discurso diferentemente do meu".

A troca de conhecimentos entre os idosos e os jovens e o diálogo intergeracional são centrais, tanto na transmissão da herança cultural e no combate à marginalização social, quanto na melhoria da qualidade da educação de jovens (GALEOTTI, 2015). Oportunidades para experimentar práticas de educação intergeracional com objetos de produção artesanal reivindicam os significados e valores autênticos do trabalho, como uma ação transformadora positiva e sustentável dos seres humanos e do mundo (ZAMAGNI, 20I5) assim como no âmbito que contribua para o crescimento e desenvolvimento da pessoa (ALESSANDRINI, 20 I2, 2004; MARGIOTTA, 20 I I).

Voltando à pesquisa apresentada aqui, o ponto nodal de análise do trabalho é compreender quais são as potencialidades de certas atividades artesanais para o desenvolvimento econômico, social e ambiental sustentável de um território. Essa questão abre uma série de reflexões que remontam a duas vertentes principais: a primeira diz respeito à capacidade de o artesanato ser considerado um setor produtivo capaz de gerar valor, não apenas econômico, mas também social; o segundo está ligado à capacidade de uma específica atividade artesanal, de representar e sustentar a identidade de um lugar e/ou 
um grupo social e o senso de pertencimento da comunidade, ao qual é uma expressão também fora de suas fronteiras.

Após apresentar os conceitos fundados no referencial teórico, essa contribuição procura ilustrar as diferentes fases da pesquisa e os resultados obtidos, finalizando com uma reflexão sobre qual poderia ser a contribuição do artesanato artístico e tradicional do sul da Itália para o desenvolvimento sustentável local.

\section{ARTESANATO TRADICIONAL, PATRIMÔNIO CULTURAL E NOVAS HABILIDADES}

Na Itália, a Lei-Quadro 433 de 8 de agosto de 1985, define a abrangência da empresa artesanal. A Lei estabelece os limites da dimensão e as formas societárias que pode assumir, bem como as modalidades de formação dos artesãos e seus órgãos representativos. Recentemente, uma rede internacional de organizações promoveu a Carta Internacional de Artesanato Artístico e Tradicional (20 I I), que representa o primeiro instrumento internacional de reconhecimento do artesanato artístico como um setor produtivo e econômico. Especificamente, as atividades que podem ser definidas como artesanato artístico, são:

a) criações, produções e obras de alto valor estético, sejam elas inspiradas em formas, modelos, decorações, estilos e técnicas tradicionais ou na história da arte, ou sejam resultado de percursos criativos individuais e linguagens expressivas e artísticas pessoais;

b) trabalho desenvolvido principalmente com técnicas manuais, com alto nível técnico e profissional, com a ajuda de equipamentos, com exceção dos processos de produção inteiramente em série; são permitidas fases de mecanização única ou automatizada de acordo com técnicas inovadoras e instrumentos tecnologicamente avançados;

c) atividades de restauração que consistam em intervenções destinadas à conservação, consolidação e restauração de bens de interesse artístico, ou pertencentes ao patrimônio arquitetônico, arqueológico, etnográfico, bibliográfico e arquivístico.

artesanato também é um dos cinco setores do patrimôno cultural imaterial incluído na Convenção da UNESCO de 2003. Aqui é reconhecida sua importância para o sentido de identidade, a proteção da diversidade cultural e da criatividade humana do ponto de vista de produção, mas acima de tudo como um elemento de identidade.

Nos últimos anos, o patrimônio cultural tem estado no centro de um debate animado sobre as políticas de desenvolvimento. Enfatizando as oportunidades e os desafios associados à sua conservação, 
salvaguarda e valorização, uma série de documentos de organismos supranacionais identificam o artesanato como uma ferramenta de coesão social, crescimento do capital humano e, portanto, um elemento fundamental para um desenvolvimento saudável da sociedade (UNESCO, UNFP \& UNDP, 20I 5).

Além disso, acredita-se que a proteção da diversidade e da expressão cultural permitem a promoção do desenvolvimento que vai além da dimensão puramente econômica e reconhece o papel chave das comunidades locais (CONSELHO DA EUROPA, 20।4, 20 I 4b; UNESCO \& UNDP, 20।3).

Ao identificar a estreita relação existente entre a criatividade vinculada ao patrimônio cultural e às esferas econômica e produtiva, o desafio de salvaguardar e promover a diversidade cultural está no "ponto de transição" - ou tensão entre dois polos opostos - entre a criação cultural e o mercado, entre valor cultural e valor de mercado (UNESCO, 20 I0). Além disso, concorda-se que o investimento em cultura e criatividade pode tornar a educação mais inclusiva e equitativa, expandindo a todos a oportunidade de aprender ao longo da vida (UNESCO, 20।4).

Finalmente, as indicações dos documentos político-estratégicos não só reconhecem o patrimônio cultural artesanal como um recurso para o desenvolvimento social e econômico, mas também como um elemento que Ihe dá o caráter de sustentabilidade (COMISSÃO EUROPÉIA, 20IOv; DÜMCKE \& GNEDOVSKY, 20I3; UN, 20I5).

Para que o artesanato seja capaz de expressar seu potencial como impulsor do desenvolvimento social, econômico e também ambiental e de um território, é necessário considerar os processos de antropização ativos nele, como uma expressão da interação complexa ente os sistemas produtivos e sócioeconômicos com o ambiente natural e cultural de referência: as interseções funcionais criadas pelas diversas esferas de interesse são capazes de gerar economias locais que transformam as áreas territoriais em áreas específicas de atividades e serviços conectados.

Aqui, as relações entre pessoas, sejam elas amigas, familiares ou formais, representam um alto valor agregado, um fator chave para investir, de modo que a especialização de cada território e seus produtos típicos, incluindo os artesãos, são capazes de gerar confiança, desencadeando a difusão horizontal do conhecimento codificado e não codificado, conectando sistemas sociais, educacionais, culturais e produtivos (BECATTINI, 1989; RULLANI, 20I0, SBORDONE, 2016).

Torna-se, portanto, essencial investir em seres humanos, não apenas em termos de desenvolvimento de capacidade técnico-produtiva, mas também e sobretudo em habilidades capazes de criar sistemas territoriais através de uma aliança entre atores diferenciados, atribuir novas funções às produções tradicionais através do envolvimento de talentos de outros setores de produção (design, informática, etc), inovação de processos e produtos, hibridização entre produtos e serviços. 


\section{UMA PESQUISA NACIONAL PARA DETECTAR O POTENCIAL DE DESENVOLVIMENTO DO ARTESANATO TRADICIONAL NO SUL DA ITÁLIA}

O objetivo da pesquisa é identificar setores de produção artesanal de excelência em seis regiões do sul da Itália como potenciais impulsionadores do desenvolvimento territorial sustentável. Mais especificamente, o objeto de indagação são as excelências produtivas artesanais capazes de, por um lado, animar o tecido social e, portanto, ser representativo da identidade cultural da comunidade local de referência. Por outro, embora estejam em risco de extinção pela falta de renovação geracional, mostram um potencial tal que possam ser considerados ${ }^{1}$.

Trata-se de uma pesquisa qualitativa destinada a identificar, analisar e selecionar a produção artesanal do sul da Itália, a partir de três dimensões fundamentais: a excelência da produção artesanal em risco de extinção ou em forte dificuldade; ser ancorada na comunidade de referência e, portanto, reconhecida como patrimônio cultural imaterial; ter potencial de desenvolvimento (Fig. I).

\section{Figura 1 - As três dimensões investigadas}

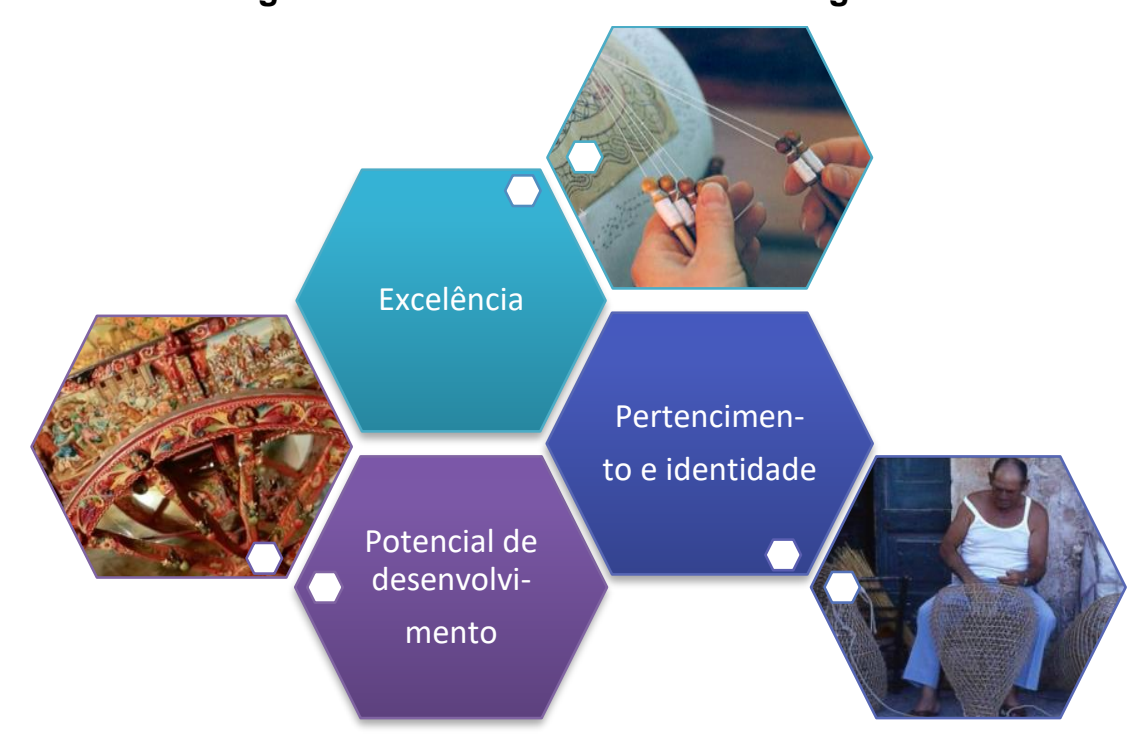

Fonte: elaboração própria (2020).

Com base nas três dimensões identificadas acima, foi construída uma matriz para a detecção, análise e seleção de produções artesanais locais, coerentes com os objetivos da pesquisa.

A matriz discrimina as dimensões investigadas nos relativos descritores.

\footnotetext{
' A pesquisa foi realizada pelo Observatório de Mestres de Artes de Florença, em nome da Fundação com o SUL. A atora coordenou, supervisionou as atividades de pesquisa até o relatório final.
} 


\begin{tabular}{|c|c|c|}
\hline DOMÍNIOS & FONTES & DESCRITORES \\
\hline \multirow{4}{*}{ 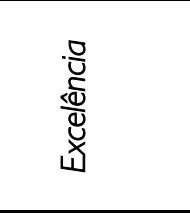 } & \multirow{4}{*}{$\begin{array}{c}\text { Marca OMA, } \\
\text { Fundação } \\
\text { Exclusiva, } \\
\text { Fundação Cologni }\end{array}$} & fases de produção, processo e produto com prevalência de processamento manual; \\
\hline & & territorialidade da matéria prima; \\
\hline & & autenticidade da produção e coerência com a tradição \\
\hline & & inovação do processo e do produto; \\
\hline \multirow{5}{*}{ 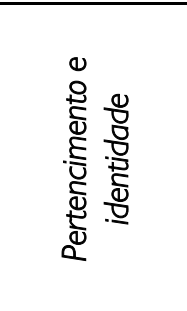 } & \multirow{5}{*}{ UNESCO, 2003} & distribuição da atividade sobre o território; \\
\hline & & função social e significado cultural para a comunidade; \\
\hline & & característica dos portadores e dos praticantes da atividade; \\
\hline & & $\begin{array}{l}\text { modalidade de transmissão de conhecimento (aprendizagem, estágios, presença de } \\
\text { cursos de formação e/ou escolas); }\end{array}$ \\
\hline & & Modalidade de manutenção do valor social e cultural (museus, festivais e outros) \\
\hline \multirow{4}{*}{ 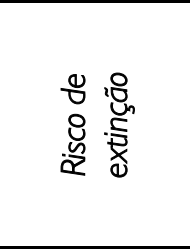 } & \multirow{4}{*}{$\begin{array}{l}\text { Instituto Italiano } \\
\text { de estatística } \\
\text { (ISTAT) }\end{array}$} & dimensão do setor (ex. Volume de negócios, n. de empregados, n. empresas); \\
\hline & & tipologia empresa (forma jurídica prevalente); \\
\hline & & tipologia de formas de trabalho (contratual prevalente); \\
\hline & & $\begin{array}{l}\text { participação em consórcios em rede, colaboração com centros de pesquisa e } \\
\text { desenvolvimento e outras entidades. }\end{array}$ \\
\hline \multirow{3}{*}{ 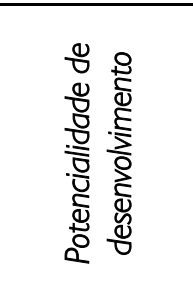 } & \multirow{3}{*}{ Ciappei, 2006} & $\begin{array}{l}\text { nível do proprietário: tipo de propriedade e suas perspectivas de sucessão familiar, extra } \\
\text { familiar, abertura para aprendizagem; }\end{array}$ \\
\hline & & $\begin{array}{l}\text { nível empreendedor: sensibilidade ao aspecto mítico simbólico, ao senso de missão, ao } \\
\text { respeito à herança do patrimônio do mestiere; }\end{array}$ \\
\hline & & $\begin{array}{l}\text { nível operativo: abertura à nova capacidade distributiva; disponibilidade à uma abertura } \\
\text { operacional sem perder os segredos da profissão; propensão à passagem de uma peça } \\
\text { ao fluxo da produção de pequenos lotes }\end{array}$ \\
\hline
\end{tabular}

Fonte: elaboração própria (2020).

A coleta de dados contribuiu para o mapeamento, a análise e a exclusão dos setores artesanais desenvolvidos nas seguintes fases.

\section{I Primeira fase de investigação: levantamento das excelências do sul da Itália em risco de extinção}

O objetivo dessa primeira fase foi identificar as produções artesanais nas seis regiões do sul da Itália que têm um caráter de excelência e que estão em risco de extinção ou em graves dificuldades. Condição de extinção ou dificuldade na transmissão de conhecimentos e habilidades artesanais. Singularidade e autenticidade da produção, relacionadas às matérias primas utilizadas, ao processo de trabalho e/ou ao produto realizado. Ligação com o território de referência.

Atividade de pesquisa realizada:

- análise da literatura de referência;

- entrevistas semiestruturadas com 16 especialistas dos setores (de um total de 24 contatados): acadêmicos, profissionais e representantes de associações das categorias; 
- entrevistas semiestruturadas com 10 partes interessadas locais (associações de categorias, instituiç̧ões e entidades locais, escolas de formação, etc.).

resultado da primeira fase da pesquisa resultou inicialmente em uma listagem de cinquenta setores do artesanato artístico e tradicional do sul da Itália, que atendem aos critérios de excelência, mas que estão em risco de extinção ou em grande dificuldade. Essa listagem foi finalmente reduzida a vinte e cinco setores, seguida de entrevistas com atores e partes locais interessadas.

\subsection{Segunda fase da pesquisa: análise dos setores selecionados a partir dos domínios indagados no estudo}

O objetivo da segunda fase foi verificar os dados coletados na primeira fase da pesquisa nos vinte e cinco setores identificados, para realizar um aprofundamento sobre as dimensões e critérios adotados no estudo e presentes na matriz previamente ilustrada. As atividades de pesquisa realizada nessa fase foram:

- entrevistas semi-estruturadas no local com 25 representantes em vários níveis dos setores identificados;

- análise dos dados coletados usando uma matriz SWOT2 $\mathrm{Sara}^{2}$ relatar dados significativos com relação aos critérios de análise escolhidos.

Os resultados dessa segunda fase foram os esquemas descritivos de cada um dos setores investigados, nos quais são relatados os dados relacionados aos descritores apresentados na matriz.

\subsection{Terceira fase: seleção de atividades artesanais de excelência que manifestam potencial para desenvolvimento}

objetivo da terceira fase foi dedicado à seleção de produções artesanais que apresentam potencial para desenvolvimento e que, portanto, podem, por meio de apoio preciso e ações sistemáticas, tornarem-se propulsores do desenvolvimento sustentável territorial. $\bigcirc$ uso de uma matriz SWOT para a sistematização e interpretação dos dados coletados nas três primeiras fases do trabalho permitiu a seleção daqueles que melhor atendiam aos critérios adotados na pesquisa. Nesta fase da pesquisa, foi realizado um estudo adicional sobre o potencial de desenvolvimento, especialmente em termos da transmissão

\footnotetext{
2 Sigla de termos em inglês que significam Strengths (forças), Weaknesses (fraquezas), opportunities (oportunidades) e threats (ameaças). Sigla em Português: FOFA. Ferramenta utilizada para elaborar planejamentos estratégicos de empresas.
} 
intergeracional do conhecimento relacionado. A atividade de pesquisa realizada nesta fase resultou em I3 entrevistas semiestruturadas com os artesãos dos setores selecionados. $\bigcirc$ resultado dessa fase foram esquemas de informações sobre o potencial de desenvolvimento de 13 experiências artesanais. A tabela abaixo (figura 3) ilustra os setores selecionados pelo trabalho de pesquisa, análise e seleção em questão, nas três fases da pesquisa.

Quadro 2 - Os setores identificados consistentes com o referencial teórico-metodológico da pesquisa

\begin{tabular}{|c|c|c|}
\hline $\begin{array}{l}\text { Divisão da produção } \\
\text { artesanal }\end{array}$ & Breve descrição da Divisão & $\begin{array}{c}\text { Região, localidade, província de } \\
\text { referência }\end{array}$ \\
\hline Bordado tradicional & $\begin{array}{l}\text { Bordado com a técnica do "quadrado" da área de } \\
\text { Oliveto Lucano, embora outras áreas da região } \\
\text { possuam técnica própria. }\end{array}$ & $\begin{array}{c}\text { BASILICATA } \\
\text { Potenza, Matera }\end{array}$ \\
\hline \multirow{3}{*}{ Trançado de fibras vegetais } & $\begin{array}{l}\text { Junco e outras fibras naturais lacustres para } \\
\text { realização de cestas }\end{array}$ & $\begin{array}{c}\text { PUGLIA } \\
\text { Salento (LE, BR, TA) }\end{array}$ \\
\hline & $\begin{array}{l}\text { Castanheira para a construção de recipientes para } \\
\text { produtos agroalimentares }\end{array}$ & $\begin{array}{c}\text { CALABRIA } \\
\text { San Giorgio Mogera (RC) }\end{array}$ \\
\hline & $\begin{array}{l}\text { Cestas de asfodel e taboa; armadilha e rede de } \\
\text { pesca em junco; cadeira empalhada }\end{array}$ & $\begin{array}{l}\text { SARDENHA } \\
\text { Área difusa }\end{array}$ \\
\hline \multirow{2}{*}{ Produção de violas } & $\begin{array}{l}\text { Produção de guitarra, violinos e outros } \\
\text { instrumentos de acordo com os ditames da tradição } \\
\text { da família De Bonis }\end{array}$ & $\begin{array}{c}\text { CALABRIA } \\
\text { Bisignano (CS) }\end{array}$ \\
\hline & $\begin{array}{l}\text { Produção de guitarra cilentana e do bandolim } \\
\text { napolitano }\end{array}$ & $\begin{array}{c}\text { CAMPANIA } \\
\text { Stio (SA) e Napoli }\end{array}$ \\
\hline \multirow[b]{2}{*}{ Produção de seda } & $\begin{array}{l}\text { Produção de jasmim para a produção de fios de } \\
\text { seda e a manufatura de produtos têxteis }\end{array}$ & $\begin{array}{c}\text { CALABRIA } \\
\text { San Floro (CZ) }\end{array}$ \\
\hline & $\begin{array}{l}\text { Produção de manufaturas em seda, reconhecidos } \\
\text { por sua altíssima qualidade, também com o uso de } \\
\text { antigos teares e desenhos Bourbon. }\end{array}$ & $\begin{array}{c}\text { CAMPANIA } \\
\text { Sete di San Leucio (CE) }\end{array}$ \\
\hline $\begin{array}{l}\text { Construção de } \\
\text { embarcações }\end{array}$ & $\begin{array}{l}\text { Construção de barcos e lanchas de Sorrento, Vietri } \\
\text { e Ischia. }\end{array}$ & $\begin{array}{c}\text { CAMPANIA } \\
\text { Sorrento e Vietri (SA), Ischia (NA) }\end{array}$ \\
\hline Tecelagem tradicional & $\begin{array}{l}\text { Técnica do "floco leccese" para a produção de } \\
\text { tapetes, tapeçarias e outros acessórios de } \\
\text { decoração, também com a utilização de teares } \\
\text { antigos. }\end{array}$ & $\begin{array}{c}\text { PUGLIA } \\
\text { Salento (BR, LE, TA) }\end{array}$ \\
\hline $\begin{array}{l}\text { Têxtil: fiação e tingimento } \\
\text { de lã de ovelha }\end{array}$ & $\begin{array}{l}\text { Fiação e tingimento de lã de ovelha com o uso de } \\
\text { corantes naturais incluídos na cadeia de produção } \\
\text { da Sardenha para realização de tapetes. }\end{array}$ & $\begin{array}{c}\text { SARDENHA } \\
\text { Difundida em todo território da } \\
\text { região }\end{array}$ \\
\hline $\begin{array}{l}\text { Construção do Carretto } \\
\text { Siciliano }\end{array}$ & $\begin{array}{l}\text { Processo de produção tradicional que envolve } \\
\text { vários mestres na fabricação dos meios típicos } \\
\text { sicilianos de reboque de equinos }\end{array}$ & $\begin{array}{c}\text { SICILIA } \\
\text { Provincia de Catania e Palermo }\end{array}$ \\
\hline
\end{tabular}

Fonte: elaboração própria (2020). 
Finalmente, é importante mencionar o método de análise e gestão dos dados coletados nas fases da pesquisa. Os dados produzidos com as entrevistas foram tratados utilizando o método de análise de conteúdo, um processo aplicado para a codificação qualitativa da informação que pode ser pensada como uma ponte entre a linguagem de pesquisa qualitativa e a linguagem da pesquisa quantitativa (BOYATZIS, 1998). Esse procedimento consiste na segmentação analítica do conteúdo em categorias e áreas conceituais através de "unidades de significado" úteis, para explicar um fenômeno (GLÄSER \& LAUDEL, 20 I3). No caso específico, a análise e sistematização das unidades ou núcleos de sentido são resultado de procedimentos de classificação da percepção dos entrevistados a partir dos descritores ilustrados na matriz.

\section{A CONTRIBUIÇÃO DO ARTESANATO TRADICIONAL PARA O DESENVOLVIMENTO SUSTENTÁVEL}

Numa primeira análise dos treze setores selecionados, é interessante notar que estes podem ser realocados em três macrocategorias, destacando qual pode ser a contribuição e as potenciais trajetórias do artesanato artístico no sul da Itália para o desenvolvimento sustentável.

A primeira macro-categoria diz respeito à sustentabilidade ambiental, a qual pode relacionar as experiências artesanais que têm como objeto, sobretudo, o trançado de fibras vegetais.

O objetivo 12 da Agenda 2030 para o Desenvolvimento Sustentável das Nações Unidas (ONU, 20I5) trata de modelos sustentáveis de produção e consumo e fornece, como subobjetivos:

- a gestão eco-compatível de todos os resíduos durante todo o seu ciclo de vida e reduzir significativamente a sua liberação no ar, na água e no solo para minimizar o seu impacto negativo na saúde humana e no ambiente;

- reduzir substancialmente a produção de resíduos através da prevenção, redução, reciclagem e reutilização.

Nesses termos, a produção de manufaturas através do trançado de fibras vegetais parece ser um elemento de importância primordial, especialmente com vistas a reduzir o uso de embalagens plásticas.

Também a restauração da cadeia de abastecimento da lã da Sardenha mostra um forte impacto ambiental, uma vez que esta matéria-prima é considerada um resíduo especial e os custos de eliminação são elevados. Paralelamente ao desenvolvimento do potencial desses setores, é necessária uma atividade de sensibilização dos consumidores, também contemplada no objetivo já mencionado.

Passando para a segunda macro-categoria, o aprimoramento do conhecimento das mulheres é um tema que pode incluir atividades tradicionais de bordados e tecelagem, atividades que muitas vezes 
dizem respeito à vestimenta doméstica, quando não há informalidade. No objetivo 5, a Agenda das Nações Unidas propõe alcançar a igualdade de gênero e empoderar todas as mulheres e meninas através, de um lado, do reconhecimento do trabalho doméstico, com o início de reformas para dar às mulheres direitos iguais de acesso aos recursos econômicos, bem como propriedade e controle da terra e outras formas de propriedade, serviços financeiros, herança e recursos naturais. $\bigcirc$ conhecimento das mulheres empregadas na produção têxtil e em bordados está em grande risco de extinção, porque são atividades que atingiram um nível empreendedor apenas em uma pequena parte, relegando a produção principalmente à esfera privada. É claro que para desenvolver essa produção é necessário recuperar as tradições, mas também redefinir as manufaturas em uma chave contemporânea. $\bigcirc$ potencial de desenvolvimento dessa produção também está ligado à restauração das cadeias produtivas.

A valorização da cultura local, finalmente, poderia ser nossa terceira macro-categoria, na qual a fabricação de instrumentos musicais e meios de transporte tradicionais (carretas sicilianas) podem ser inseridas. Preservar o uso dos costumes locais não é apenas uma questão de folclorismo, mas um elemento para tornar as cidades e os assentamentos humanos inclusivos, seguros, duradouros e sustentáveis, fortalecendo os esforços para proteger e salvaguardar o patrimônio cultural e natural do mundo.

Além disso, o valor intrínseco dessas produções representa não apenas um meio de manter e valorizar a diversidade cultural de nosso país, mas podem também contribuir para a sustentabilidade econômica, social e ambiental das ações de desenvolvimento local.

\section{CONSIDERAÇÕES FINAIS}

Além de identificar alguns setores do artesanato artístico que estão em risco de extinção, mas que evidenciam potencialidade de desenvolvimento, outro resultado da pesquisa foi a preparação e aplicação de uma matriz de múltiplos critérios para identificar e analisar o potencial econômico e social das produções artesanais. De fato, a maior parte das pesquisas dedicadas ao artesanato adota uma perspectiva monodisciplinar, o que permite compreender apenas parcialmente os pontos fortes e/ou pontos fracos expressos por determinadas produções. Na Itália, pesquisas que destacam a dimensão educacional e formativa intergeracional são raras ou quase inexistentes. A maioria das investigações e reflexões é desenvolvida no campo das políticas econômicas e de desenvolvimento (D'AGOSTINO, FERRITTI, 20 I6). Nesse sentido, é interessante notar que algumas dimensões educacionais são mencionadas entre os critérios utilizados.

Um primeiro elemento diz respeito à existência de oportunidades de treinamento sobre a atividade artesanal específica entre as gerações, através de dispositivos educacionais, como estágios e outros, mas 
também a capacidade do empreendedor de aproveitar essas mesmas oportunidades para transmitir seu próprio saber e saber fazer.

Um segundo elemento, diz respeito às habilidades que o setor de produção investigado expressa, que afetam as várias etapas da produção e a capacidade de inovar o processo e/ou produto. Esse aspecto é decisivo para projetar ações capazes de enfrentar o perigo de extinção da produção considerada de excelência

Consequentemente, o estudo revelou as necessidades de formação daqueles que trabalham nesses setores de artesanato e daqueles que trabalham para aprimorá-los para o desenvolvimento local. Nessa perspectiva, a pesquisa deve ser considerada a primeira fase de um processo mais direto para experimentar ações inovadoras, para apoiar a produção artesanal e o desenvolvimento social em áreas deprimidas ou marginais.

Os setores artesanais identificados foram objeto de um "convite à apresentação de propostas" emitido pela Fundação com o $\mathrm{Sul}^{3}$ e dirigido a organizações do terceiro setor. $\bigcirc$ objetivo é a criação de empreendimentos sociais, a partir da inovação e refuncionalização de processos tradicionais e/ou produtos artesanais, por meio de ações de incubação de empresas que envolvem mestres de artes e jovens talentos. A Fundação selecionou três projetos, via edital: um dedicado à construção de bicicletas de vagão, decoradas com a iconografia típica das carroças sicilianas proposto por um grupo de jovens designers de Palermo; outro sobre a produção de artefatos têxteis, como o uso da técnica "fiocco leccese" em Puglia e um terceiro sobre a utilização de materiais impressos em 3D para a construção de bandolins napolitanos em Scampia, um distrito de Nápoles com alto risco social. Os projetos, que começarão em janeiro de 2020, envolverão mestres de artes, jovens talentos e setores menos favorecidos da população (jovens em busca de ocupação, mulheres desempregadas e ex prisioneiros). Todos os três estão localizados em áreas altamente privadas e com necessidade de ações de desenvolvimento e coesão social. Em cada um dos três projetos, serão testadas ações e dispositivos inovadores de treinamento intergeracional, vinculados à refuncionalização produtiva, inclusão social e criação de empresas nos territórios de referência. (FEDERIGHI, 20I2)

\section{REFERÊNCIAS}

ALESSANDRINI, G. La pedagogia del lavoro. Questioni emergenti e dimensioni di sviluppo per la ricerca e la formazione. Education Sciences \& Society, 3(2), 55-72, 2012.

\footnotetext{
${ }^{3}$ Fundação com o Sul é uma fundação de origem bancária que financia projetos de inovação social para o desenvolvimento na região do sul da Itália (https://www.fondazioneconilsud.it). No caso da iniciativa sobre a extinção das atividades artesanais de excelência, a Fundação disponibilizou um milhão de euros.
} 
ALESSANDRINI, G. Pedagogia delle risorse umane e delle organizzazioni. Milano: Guerini e associati editore, 2004.

BASCHIERA, B., DE LUIGI, R., LUPPI, E. Educazione intergenerazionale. Prospettive, progetti e metodologie didattico-formative per promuovere la solidarietà fra le generazioni Milano: Franco Angeli, 2014 .

BATESON, G. Mind and Nature, a Necessary Unity. NY: Dutton, 1979.

BATESON, G. Steps to an Ecology of Mind. London: Paladin Books, 1973.

BECATTINI, G. Modelli locali di sviluppo. Bologna: II Mulino, 1989.

BOYATZIS, R.E. Transforming qualitative information: Thematic analysis and code development. Thousand Oaks, London, New Delhi: Sage Publications, 1998.

BRONFENBRENNER, U. The Ecology of Human Development. Harvard University Press, Cambridge, MA, 1979.

COSTA, M. Futuro artigiano: I'agire generativo tra comunità e network. Venezia: Quaderni di ricerca sull'artigianato, CGIA, 20II.

Council of Europe. Conclusions on cultural heritage as a strategic resource for a sustainable Europe, 20 I 4a. https://www.consilium.europa.eu/uedocs/cms_data/docs/pressdata/en/educ/I 42705.pdf

COUNCIL OF THE EUROPEAN UNION. Work Plan of Culture 20I5-20I8, 20 I 4b.

DUMCKE, C.; GNEDOVSKY, M. The Social and Economic Value of Cultural Heritage: literature review. EENC Paper, 20 13. http://www.interarts.net/descargas/interarts2557.pdf

European Commission. Towards an integrated approach to cultural heritage for Europe, 2014. http://ec.europa.eu/culture/ibrary/publications/2014- heritage communication en.pdf

FEDERIGHI P., I processi di apprendimento nel lavoro. In: FEDERIGHI, Paolo, CAMPANILE, Gabriella, GRASSI, Cinzia. II modello dell'Embedded Learning nelle PMI. Pisa: Edizioni ETS, 20 I2, pp. 43-47.

FREIRE P. Pedagogia dell'autonomia. Saperi necessari per la pratica educativa, Torino: Ega-Edizioni Gruppo Abele, 2004.

GALEOTTI G. Connecting Generations (Congenial) Learning Partnership: creating common practices and a shared visions in intergenerational education. In GALOETTI G. (Org). CONGENIAL. Connecting Generations: Intergenerational learning for a more inclusive Europe. Firenze: Via Laura, 20 I 5.

GALEOTTI, G., The educational valorisation of traditional knowledge. An intervention-research with tuleros, Mayan artisans of Atitlán Lake in Guatemala, In: PINTON S., ZAGATO L. (Org.). Cultural Heritage. Scenarios 20 15-20 I 7. Venezia: Edizioni Ca' Foscari Digital Publishing, 2018.

GHERARDI, S. Breve storia di un concetto in viaggio: dalle comunità di pratiche alle pratiche di una comunità. Studi Organizzativi, v. 10, n. I, pp. 49 -72, 2008. 
GLÄSER, J.; LAUDEL, G. Life With and Without Coding: Two Methods for Early-Stage Data Analysis. Qualitative Research Aiming at Causal Explanations, Forum: Social qualitative research», 14, n. 2, art. 5 , 2013.

INGOLD, T. Making. Milano: Raffaele Cortina editore, 2019.

INGOLD, T. Ecologia della culturaì. Roma: Meltemi, 2004.

MARGIOTTA, U. Per una nuova pedagogia dell'età adulta. Pedagogia Oggi, vol. I-2, 67-82, 20 I l .

LAVE, J.; WENGER, E. Situated Learning: Legitimate Peripheral Participation, Cambridge: Cambridge University Press, 1991.

RULLANI, E. Modernità sostenibile. Idee, filiere e servizi per uscire dalla crisi. Venezia: Marsilio Editore, 2010.

SENNETT, R. The Craftsman. New Haven Yale: University Press New Haven.

Sbordone, M.A. (20 I6). Design Networking. Sistemi locali di economie collaborative. Impresa sociale, numero 8, 2008.

UNITED NATION - UN. Agenda 2030 per lo sviluppo sostenibile, 2015.

https://sustainabledevelopment.un.org/?menu $=1300$

UNESCO. Convention for the Safeguarding of the Intangible Cultural Heritage, 2003.

http://portal.unesco.org/en/ev.php-

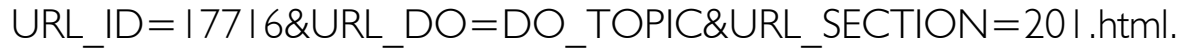

UNESCO. Convention on the Protection and Promotion of the Diversity of Cultural Expressions, 2005. http://portal.unesco.org/en/ev.php-

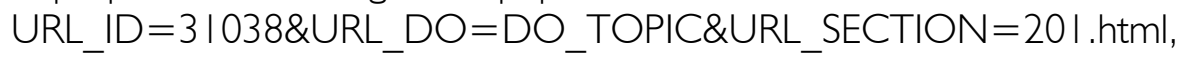

UNESCO. World Report. Investing on Cultural Diversity and Intercultural Dialogue, 2010.

http://www.unesco.org/ibrary/PDF/Diversity

UNESCO. Culture, Creativity and Sustainable Development. Research, Innovation, Opportunities, Florence Declaration, 2014.

UNESCO, UNDP. Creative Economy Report. Widening local development pathways, 2013. http://www.unesco.org/culture/pdf/creative-economy-report-20I3.pdf.

UNESCO, UNFPA, UNDP, Post-20I 5 Dialogues on Culture and Development report, 2015. http://unesdoc.unesco.org/images/0023/002322/232266E.pdf.

VASSALLO, N. Teoria della conoscenza, Roma-Bari: Laterza, 2003.

WENGER, E. HOW WE LEARN. COMMUNITIES OF PRACTICE: THE SOCIAL FABRIC OF A LEARNING ORGANIZATION, HEALTHC FORUM J., JUL-AUG, 39(4), PP. 20-26, 1996.

ZAMAGNI, S. Giustizia sociale, lavoro, bene comune. In C. Danani (eds.). L'umano tra cura e misura. Milano: Vita e Pensiero, 2015. 